\title{
Transverse Instability of Rogue Waves
}

\author{
Mark J. Ablowitz ${ }^{1}$ and Justin T. Cole ${ }^{2}$ \\ ${ }^{1}$ Department of Applied Mathematics, University of Colorado, Boulder, Colorado 80309, USA \\ ${ }^{2}$ Department of Mathematics, University of Colorado, Colorado Springs, Colorado 80918, USA
}

(Dated: September 14, 2021)

\begin{abstract}
Rogue waves are abnormally large waves which appear unexpectedly and have attracted considerable attention, particularly in recent years. The one space, one time $(1+1)$ nonlinear Schrödinger equation is often used to model rogue waves; it is an envelope description of plane waves and admits the so-called Pergerine and Kuznetov-Ma soliton solutions. However, in deep water waves and certain electromagnetic systems where there are two significant transverse dimensions, the $2+1$ hyperbolic nonlinear Schrödinger equation is the appropriate wave envelope description. Here we show that these rogue wave solutions suffer from strong transverse instability at long and short frequencies. Moreover, the stability of the Peregrine soliton is found to coincide with that of the background plane wave. These results indicate that, when applicable, transverse dimensions must be taken into account when investigating rogue wave pheneomena.
\end{abstract}

In recent years researchers have studied a class of large amplitude waves that were previously relegated to folklore: giant water waves appearing out of nowhere causing extreme damage to, and even loss of, maritime vessels. The first verified measurement of an extreme water wave was Jan 1, 1995 on the Draupner platform in the North Sea where a $25.6 \mathrm{~m}(84 \mathrm{ft})$ wave was observed [1]; much larger than the background wave field. Subsequently, rogue waves events have been observed in several laboratory settings such as wave tanks [2, 3], nonlinear optics [4 [6], superfluid helium [7], and plasmas [8].

The 1+1 dimensional nonlinear Schrödinger (NLS) equation is a well-known model used to describe the envelopes of generic nonlinear plane wave solutions in one spatial dimension (corresponding to the direction of propagation) and one temporal dimension [9]. In water waves, the nonlinear plane wave was found by G. Stokes in the mid-1800's [10]. Importantly, the underlying NLS equation admits special large amplitude "rogue wave" solutions including the Peregrine [11] and Kuznetov-Ma (KM) solitons [12, 13]. However, a more accurate wave envelope approximation of deep water waves takes into account the transverse variation; this is the $2+1$ dimensional NLS equation -see Eq. (11) below.

A physically significant regime in deep water waves corresponds to small surface tension, which is described by the $2+1$ 'hyperbolic' NLS (HNLS) equation [14]. Another important application of the HNLS equation is spatiotemporal electromagnetc wave propagation in media with normal dispersion cf. [15, 16]. While other more sophisticated models do exist, NLS models can suggest possible mechanisms that will be relevant in applications even where NLS might not be the optimal model. We note that other $2+1$ water wave models are frequently used in the study of rogue waves, such as crossing states [17 19], but we do not discuss those phenomena here. The KM and Peregrine solitons are known to be unstable in $1+1$ dimensions for scalar [20, 21] and vector systems [22], but corresponding stability analysis in $2+1$ dimensions has not been carried out. In this case we show that both of these solitons suffer from transverse instability.

Transverse instability of nonlinear solutions is an im- portant and well-known effect, e.g., the transverse instability of localized solitons by long wavelengths was first found in the mid 1970s 23]. By employing Floquet theory we show that there are instabilities at arbitrarily small transverse wavelength scales with finite growth rates. Indeed, it has been long known that the Stokes plane wave solution of the HNLS equation is unstable to transverse variations 24 26]. With this observation it is perhaps to be expected that the Peregrine and KuznetovMa solitons, which at large distances limit to the plane wave, will also suffer from this serious instability. But what is remarkable is the similarity of the instability profiles between the plane waves and KM/Peregrine solitons, particularly at high transverse frequencies. Furthermore, in the hyperbolic case these rogue waves are found to have an instability region for all transverse wave numbers, as opposed to a finite region instability like the elliptic version.

We note a major difference between the instabilities of the elliptic and hyperbolic NLS equations. The elliptic version does not have growth rates at arbitrarily small transverse wavelengths; this instability, first found in one dimensional water waves 27], is often termed modulational instability [28]. Moreover, the elliptic-focusing NLS equation with cubic nonlinearity can suffer from collapse in finite time [29] whereas, to our knowledge, it is not known if the hyperbolic version exhibits finite time wave collapse. Numerical evidence that Peregrine solitons can collapse in the elliptic NLS equation was found in 30 .

The governing NLS equation is introduced and the relevant soliton solutions are presented below. The stability of plane wave solutions and KM solitons are calculated: the former by analytic methods and the latter numerically through Floquet theory. Direct numerical simulations confirm our stability findings. We conclude that a full $2+1$ study is important for rogue wave systems when there are two significant transverse dimensions.

Consider a plane wave envelope $u(x, y, t)$ propagating through a nonlinear dispersive media in a preferred $x$ direction. Going to a translating coordinate frame moving at the group velocity and then changing variables 
leads to the dimensionless NLS equation

$$
i u_{t}+u_{x x}+s_{1} u_{y y}+2 s_{2}\left(|u|^{2}-u_{0}^{2}\right) u=0,
$$

where $s_{1}, s_{2}= \pm 1$. As $|x| \rightarrow \infty, u$ approaches a constant, $u_{0}$; without loss of generality, we set $u_{0}=1$. In water waves, the sign of the coefficients depends on the surface tension-cf. Fig. 4.15 in [14]. For small surface tension (ST) we have: $s_{1}=-1, s_{2}=1$, termed hyperbolic; for moderate ST: $s_{1}=1, s_{2}=-1$, called ellipticdefocusing; and at sufficiently large ST: $s_{1}=s_{2}=1$, termed elliptic-focusing. The hyperbolic-focusing $\left(s_{1}=\right.$ $\left.-1, s_{2}=1\right)$ and hyperbolic-defocusing $\left(s_{1}=-1, s_{2}=\right.$ -1 ) equations are equivalent up to conjugation and exchange of $x$ and $y$ in (1). As a result, they will yield similar stability results below and we shall simply refer to the "hyperbolic-focusing" signs as the "hyperbolic" NLS equation from here on.

There are two well-known $1+1$ soliton solutions of the focusing NLS equation $\left(s_{2}=1\right)$ we shall focus on. The first is the KM soliton, which is periodic in $t$ and localized in $x$, given by

$$
\begin{aligned}
& u_{\mathrm{KM}}(x, t)= \\
& \quad \frac{\cosh c_{-} x+\frac{1}{2 c_{+}}\left(c_{+}^{2}+c_{-}^{2}\right) \sin s-i c_{-} \cos s}{\cosh c_{-} x+\frac{2}{c_{+}} \sin s},
\end{aligned}
$$

where $s=c_{+} c_{-} t-\pi / 2, c_{ \pm}=Z \pm 1 / Z, Z>1$ [see Fig. 1(a)]. The parameters are chosen so that the soliton peak occurs at $x=0$ and the largest (smallest) magnitude $\left|u_{\mathrm{KM}}(0, t)\right|$ at times $t=n T\left(\frac{(2 n+1) T}{2}\right)$ for $n \in \mathbb{Z}$, with period $T=2 \pi /\left(Z^{2}-1 / Z^{2}\right)$. As $Z \rightarrow 1$, this solution approaches the Peregrine soliton

$$
u_{\mathrm{P}}(x, t)=\frac{4 x^{2}-16 i t+16 t^{2}-3}{4 x^{2}+16 t^{2}+1},
$$

which is localized in both $x$ and $t$ since $T \rightarrow \infty$ [see Fig. 1(b)]. At large distances both solutions approach a plane wave, i.e., as $|x| \rightarrow \infty, u_{\mathrm{KM}}, u_{\mathrm{P}} \rightarrow \exp (2 i t)$. In terms of the inverse scattering transform, we assume that as $|x| \rightarrow \pm \infty,|u|$ tends to unity sufficiently fast. This class includes the KM soliton (with exponentially fast decay), but not the Peregrine soliton which decays algebraically fast cf. 31].

We now study the stability of these solitons to transverse perturbations. Consider the two-dimensional perturbation

$$
u(x, y, t)=\widetilde{u}(x, t)+w(x, y, t)
$$

where $\widetilde{u}(x, t)$ is a solution of (1) and the function $w$ has small magnitude. Linearizing (11) about the solution gives

$$
i w_{t}+w_{x x}+s_{1} w_{y y}+2 s_{2}\left[\left(2|\widetilde{u}|^{2}-1\right) w+\widetilde{u}^{2} w^{*}\right]=0,
$$

where $w^{*}$ is the complex conjugate of $w$. We look for Fourier solutions of the form

$$
w(x, y, t)=w_{+}(x, t) e^{i \ell y}+w_{-}(x, t) e^{-i \ell y} .
$$

(a)

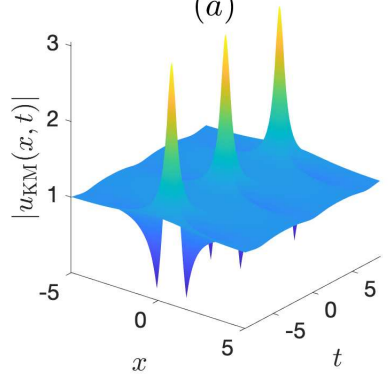

(b)

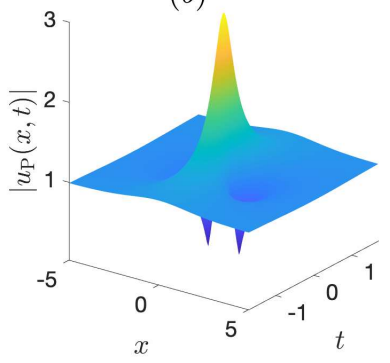

FIG. 1. Magnitude evolutions of the (a) Kuznetzov-Ma soliton in (2) with $Z=1.25$ and (b) Peregrine soliton in (3). $(a)$

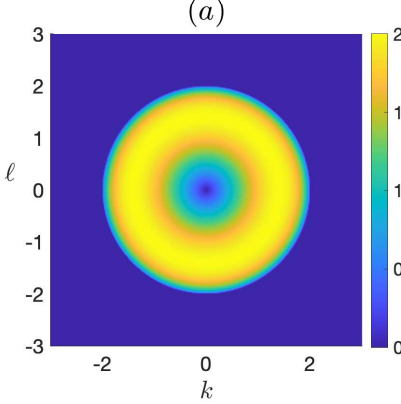

(b)

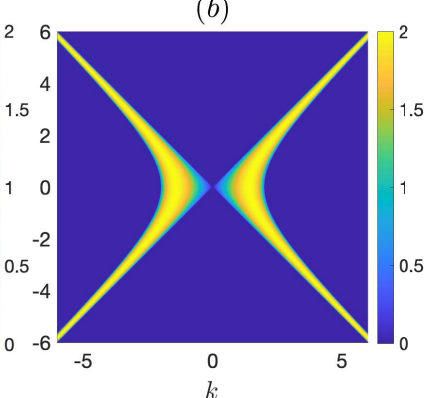

FIG. 2. Plane wave instability (MI): (a) Elliptic-focusing $\left(s_{1}=1=s_{2}\right)$ and (b) hyperbolic $\left(s_{1}=-1, s_{2}=1\right)$ instability regions and growth rates. Plotted is $|\operatorname{Im}\{\lambda\}|$ for $\lambda(k, \ell)$ in (77).

Setting the coefficients of $e^{ \pm i \ell y}$ to zero yields

$i \partial_{t} w_{+}+\left[\partial_{x}^{2}-s_{1} \ell^{2}+2 s_{2}\left(2|\widetilde{u}|^{2}-1\right)\right] w_{+}+2 s_{2} \widetilde{u}^{2} w_{-}^{*}=0$

$i \partial_{t} w_{-}^{*}-\left[\partial_{x}^{2}-s_{1} \ell^{2}+2 s_{2}\left(2|\widetilde{u}|^{2}-1\right)\right] w_{-}^{*}-2 s_{2}\left(\widetilde{u}^{*}\right)^{2} w_{+}=0$.

We consider two cases: (a) modulational instability (MI) of a plane wave where $\widetilde{u}(x, t)=1$ and $w_{ \pm}(x, t)=$ $\alpha_{ \pm} \exp [ \pm i(k x-\lambda t)]$; and $(b)$ transverse instability of the KM soliton where $\widetilde{u}(x, t)=u_{\mathrm{KM}}(x, t)$ and $w_{ \pm}$is a Floquet mode which satisfies

$$
\left(\begin{array}{l}
w_{+} \\
w_{-}^{*}
\end{array}\right)(x, t+T)=e^{-i \lambda(\ell) T}\left(\begin{array}{l}
w_{+} \\
w_{-}^{*}
\end{array}\right)(x, t)
$$

for Floquet exponent $\lambda(\ell)$ 32].

For the Peregrine soliton i.e. $\widetilde{u}=u_{\mathrm{P}}$, the form of the eigenfunction $w$ is unclear since the coefficients in (5) are localized in both $x$ and $t$. Instead we take an indirect approach: since the KM soliton approaches the Peregrine soliton as $Z \rightarrow 1$, we expect that the stability of KM will also approach that of Peregrine.

For plane wave solutions, stability system (5) has constant coefficients. Looking for plane wave eigenmodes yields the eigenvalues

$$
\lambda^{2}=\left(k^{2}+s_{1} \ell^{2}\right)\left(k^{2}+s_{1} \ell^{2}-4 s_{2}\right)
$$


which correspond to linear instability when $\operatorname{Im}\{\lambda\} \neq 0$. When $\ell=0$ we recover the classic one-dimensional MI result: unstable for $s_{2}=1$ (focusing) and stable when $s_{2}=-1$ (defocusing) cf. [28.

The two-dimensional problem corresponds to $\ell \neq 0$ where the plane wave is stable in the elliptic-defocusing case: $s_{1}=1, s_{2}=-1$; and in the elliptic-focusing case

$$
s_{1}=s_{2}=1 \text { instability occurs when: } 0<k^{2}+\ell^{2}<4 \text {. }
$$

The punctured disk region of instability is shown in Fig. 2(a) with maximal instability of $\lambda_{\max }= \pm 2 i$ along the circle $k^{2}+\ell^{2}=2$. This is reminiscent of the classic $1 \mathrm{D} \mathrm{MI}$ result in that there is a band-limited region in the Fourier plane corresponding to unstable wavenumbers. Outside this long wavelength region, the linear stability analysis above does not predict any exponential growth.

In the hyperbolic equation (which corresponds to deep water waves with small ST)

$s_{1}=-1, s_{2}=1$ instability occurs when $\ell^{2}<k^{2}<\ell^{2}+4$.

The hyperbolic region of instability is shown in Fig. 2(b) with largest instability of $\lambda_{\max }= \pm 2 i$ along the hyperbola $k^{2}-\ell^{2}=2$. We see that there is instability for arbitrarily small wavelengths i.e. large $|k|,|\ell|$, with a finite growth rate when $k^{2}=\ell^{2}+\gamma_{1}^{2}$ :

$$
\lambda^{2}=\gamma_{1}^{2}\left(\gamma_{1}^{2}-4\right), \quad 0<\gamma_{1}^{2}<4 .
$$

Since the KM and Peregrine soliton limit as $x \rightarrow \pm \infty$ is this unstable plane wave, we expect serious instability will also ensue for these solitons. This is confirmed by numerical calculations below.

The hyperbolic stability problem (5) is next solved with KM coefficients (2) at different transverse wavenumbers using an exponential time-differencing integrator [33]. We point out that the $1+1$ results in [20] correspond to the $\ell=0$ case. The imaginary (unstable) part of the Floquet exponents is shown in Fig. 3 (red curves). Also shown are the unstable eigenvalues for plane wave solutions (blue curves). As $Z \rightarrow 1$, the decay rate approaches that of the Peregrine soliton; as a result, the computational window used to solve the problem is widened to ensure effectively constant boundary conditions for large $|x|$. One consequence of this is additional unstable modes and faster apparent rapidity of the humps in Fig. 3 [34].

Overall, there is remarkable overlap between the soliton and plane wave instabilities, especially at large $\ell$. An important observation is that KM solitons, like the background plane waves, are transversely unstable at high frequencies. As $Z \rightarrow 1$, the spectrum of the KM soliton is approaching that of the plane wave; consisting of rapidly varying periodic hump-like structures bounded by 2 . It is remarkable that the stability spectrum of a Peregrine soliton is the same as that of a plane wave. Our results suggest that the instability of rogue waves for large transverse wave numbers can be conjectured based on the properties of the plane wave background.

The stability of the KM solitons in the elliptic-focusing NLS equation were also computed (strong ST regime in
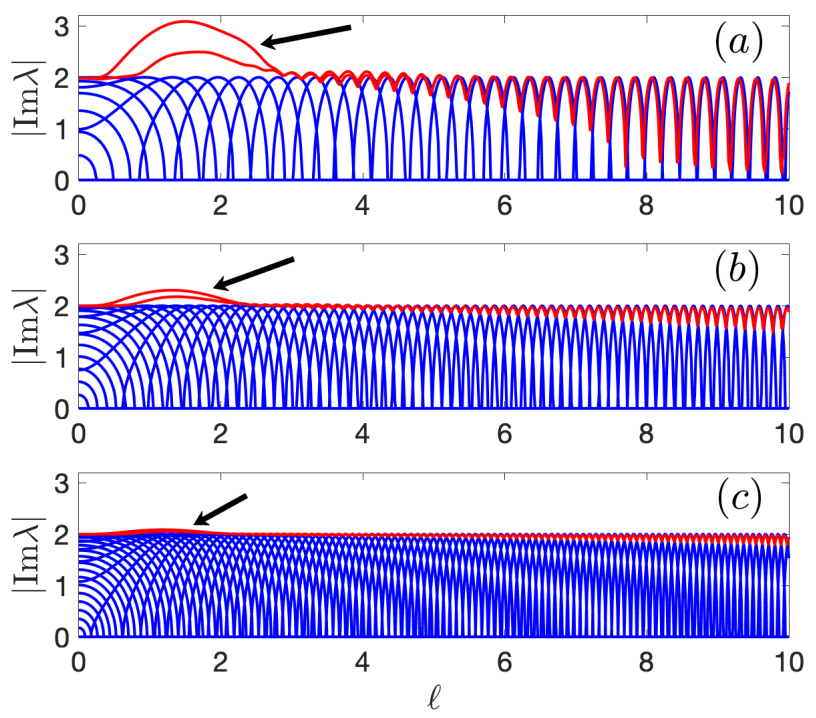

FIG. 3. Hyperbolic $\left(s_{1}=-1, s_{2}=1\right)$ transversely unstable modes; blue curves: unstable plane wave modes as function of transverse wavenumber $\ell$. red curves: two largest unstable $\mathrm{KM}$ modes for (a) $Z=2$, (b) $\mathrm{Z}=1.5$, (c) $\mathrm{Z}=1.25$. Arrows point at the KM soliton instability curves. At large $\ell$ values, the plane wave and KM soliton instability curves nearly overlap.
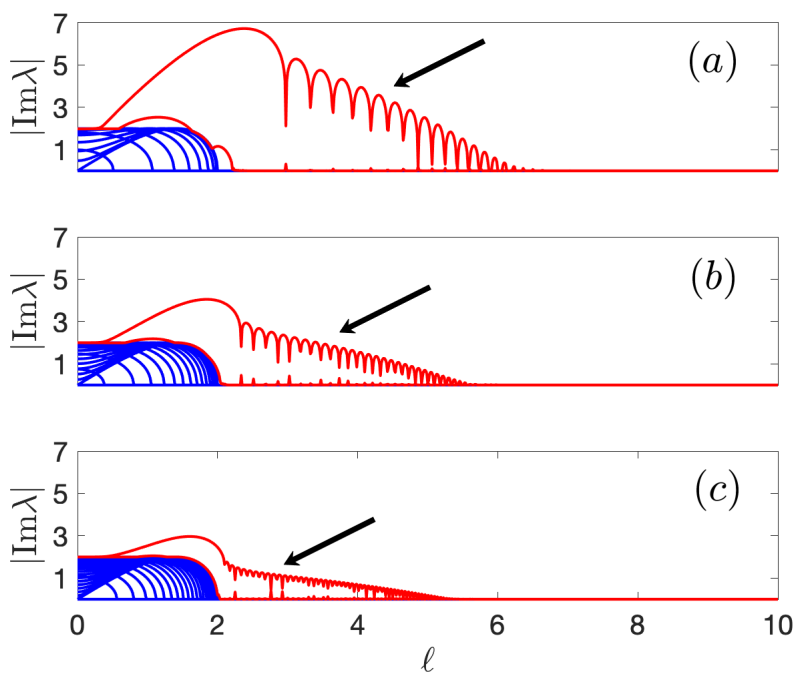

FIG. 4. Elliptic-focusing $\left(s_{1}=1, s_{2}=1\right)$ transversely unstable modes; blue curves: unstable plane wave modes as function of transverse wavenumber $\ell$. red curves: two largest unstable KM modes for (a) $Z=2$, (b) $\mathrm{Z}=1.5$, (c) $\mathrm{Z}=1.25$. Arrows point at the KM soliton instability curves.

deep water waves). The results, shown in Fig. 4, exhibit typical instability at long wavelengths. As $Z \rightarrow 1$, the interval of transversely unstable KM modes shrinks and the maximum magnitude over all $\ell$ tends to decrease and approach 2. Similar to the hyperbolic case, the soliton instability appears to be approaching that of the plane 

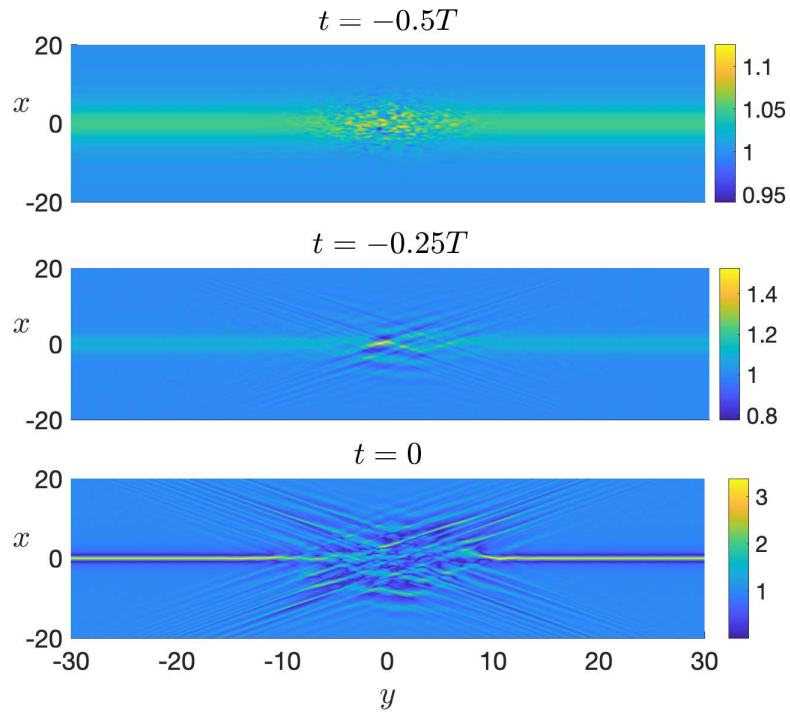

FIG. 5. Snapshots of the perturbed KM soliton $\left(\widetilde{u}=u_{\mathrm{KM}}\right)$ evolution $(Z=1.25, T \approx 6.81)$ for hyperbolic $\operatorname{NLS}\left(s_{1}=\right.$ $\left.-1=-s_{2}\right)$. Shown is $|u(x, y, t)|$ seeded with a $10 \%$ localized perturbation at $\bar{t}=-T / 2$.

wave, though not as dramatically. Unlike the hyperbolic case, there is no instability indicated for large $\ell$. As indicated in Fig. 3 and Fig. 4, we see that in the hyperbolic case there is an instability regime for all transverse wave numbers whereas the instability zone for the elliptic case has finite size.

Finally, we examine the evolution of transversely perturbed rogue waves. Consider a perturbed solution at time $t=\bar{t}$ of the form

$$
u(x, y, \bar{t})=\widetilde{u}(x, \bar{t})+w(x, y),
$$

where $\widetilde{u}(x, \bar{t})$ is a line soliton solution and $w(x, y)$ is a normally distributed random function modulated by a slowly decaying Gaussian function. The peak magnitude of $w$ is taken to be $10 \%$ that of $\widetilde{u}(x, \bar{t})$ and typically we take $\bar{t}=-T / 2$, where the KM soliton has minimal magnitude [34].

First, consider the KM soliton at $Z=1.25$; we recall it has the instability spectrum shown in Fig. 3(c). By the time the soliton reaches its peak magnitude at $t=0$, the soliton has disappeared near the perturbation and an ' $\mathrm{x}$ wave' has started to develop - see Fig. [5] We point out that this is the result of a perturbation whose magnitude is roughly $3 \%$ the maximum soliton peak.

Even though the solitons are unstable at large wavenumbers, it corresponds to a suitable combination of wavenumbers, namely, it must be those Fourier modes in the hyperbolic instability region shown in Fig. 2(b). Applying a perturbation with no $x$-dependence $(k=0$ modes) does not yield instability (see [35]). This is a subtle difference from other types of transverse instability.

In further support of our analytical expectations that the stability of the Peregrine soliton is well-approximated
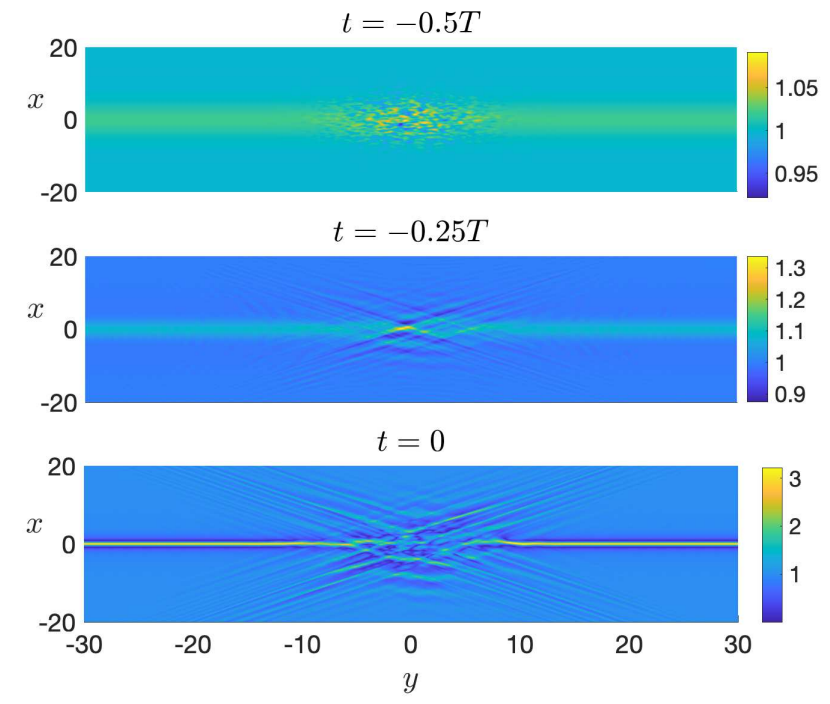

FIG. 6. Snapshots of the perturbed Peregrine soliton $\left(\widetilde{u}=u_{\mathrm{P}}\right)$ evolution for hyperbolic NLS $\left(s_{1}=-1=-s_{2}\right)$. Shown is $|u(x, y, t)|$ seeded with a $10 \%$ localized perturbation at $\bar{t}=$ $-T / 2$ for $T \approx 6.81$.

by a limiting KM soliton, we perform the following simulation, highlighted in Fig. 6. Well before the maximum peak occurs, the Peregrine soliton is perturbed by the form given in (8). At the maximum focusing point, the line soliton has again broken apart near the region where the perturbation is applied. We point out that perturbing the soliton earlier will result in earlier onset of the instability and degradation of the mode.

As a final note, these results for the hyperbolic NLS differ immensely from those of elliptic-focusing NLS. KM solitons are transversely unstable at long wavelength perturbations in the elliptic-focusing NLS equation and, also importantly, perturbed solutions can collapse in finite time. Indeed, perturbations of the sort in (8) indicate blow up occurs. Numerical simulations illustrating this are given in [35].

In conclusion, transverse stability of rogue waves was studied in the NLS equation. Linear stability of the Kuznetsov-Ma soliton was computed via Floquet theory. Since the Kuznetsov-Ma soliton approaches the Peregrine soliton, it is anticipated that its instability features will also approach those of Peregrine. Indeed, the stability of the Peregrine soliton was found to coincide with that of plane waves. Importantly, in the hyperbolic case this leads to instability at arbitrarily high frequencies from wavenumbers in a hyperbolic region in the spectral plane. Direct numerical solutions confirm that appropriately perturbed solitons are transversely unstable. Based on the above considerations it is natural to expect that two dimensional perturbations can annihilate the KM or Peregrine soliton solutions. 


\section{ACKNOWLEDGEMENTS}

This work was partially supported by AFOSR under Grant No. FA9550-19-1-0084 and NSF under Grants DMS-1712793 and DMS-2005343.

[1] S. Haver, Rogue Waves Workshop,1, (2004).

[2] A. Chabchoub, N.P. Hoffmann, N. Akhmediev, Phys. Rev. Lett., 106204502 (2011).

[3] A. Chabchoub, S. Neumann, N.P. Hoffman, N. Akhmediev, J. Geophys. Resch: Oceans,117 C11 (2013).

[4] D.R. Solli, C. Ropers, P. Koonath, B. Jalali, Nature, 450, 1054 (2007).

[5] B. Kibler, J. Fatome, C. Finot, G. Millot, F. Dias, G. Genty, N. Akhmediev, and J. M. Dudley, Nat. Phys., 6, 790 (2010).

[6] C. Lecaplain, Ph. Grelu, J. M. Soto-Crespo, and N. Akhmediev, Phys. Rev. Lett., 108, 233901 (2012).

[7] A. N. Ganshin, V. B. Efimov, G. V. Kolmakov, L. P. Mezhov-Deglin, and P. V. E. McClintock, Phys. Rev. Lett., 101, 065303 (2008).

[8] H. Bailung, S. K. Sharma, and Y. Nakamura, Phys. Rev. Lett., 107, 255005 (2011).

[9] J. Yang, Nonlinear Waves in Integrable and Nonintegrable Systems (SIAM, Philadelphia, 2010).

[10] G.G. Stokes, Trans. Camb. Phil. Soc., 8, 441, (1847).

[11] D.H. Peregrine, J. Austr. Math. Soc. Ser. B, 25, 16, (1983).

[12] E.A. Kuznetsov, Sov. Phys. Dokl., 22 507, (1979).

[13] Y.-C. Ma, Stud. Appl. Math., 6043 (1979).

[14] M.J. Ablowitz, H. Segur Solitons and the Inverse Scattering Transform, SIAM (1981).

[15] M.J. Ablowitz, Nonlinear Dispersive Waves, Cambridge Univ. Pr. (2011).

[16] P. Di Trapani, G. Valiulis, A. Piskarskas, O. Jedrkiewicz, J. Trull, C. Conti, and S. Trillo, Phys. Rev. Lett., 91 093904, (2003).

[17] M. Onorato, A.R. Osborne, M. Serio, Phys. Rev. Lett. 96 014503, (2006).

[18] M.J. Ablowitz, T.P. Horikis, Phys. Fluids 27, 012107, (2015).
[19] M.L. McAllister, S. Draycott, T.A.A. Adcock, P.H. Taylor, T.S. van den Bremer, J. Fluid Mech. 860, 767 (2019).

[20] J. Cuevas-Maraver, P. G. Kevrekidis, D. J. Frantzeskakis, N. I. Karachalios, M. Haragus, and G. James, Phys. Rev. E 96, 012202 (2017).

[21] A. Calini, C.M. Schober, and M. Strawn, Appl. Num. Math. 141, 36 (2019).

[22] F. Baronio, M. Conforti, A. Degasperis, S. Lombardo, M. Onorato, and S. Wabnitz, Phys. Rev. Lett. 113, 034101 (2014).

[23] V.E. Zakharov, A.M. Rubenchik, Sov. Phys. JETP 38 494 (1974).

[24] V.E. Zakharov, Sov. Phys. J. Appl. Mech. Tech. Phys., 4 190, (2009).

[25] D.J. Benney and G.J. Roskes, Stud. Appl. Math. 48377 (1969).

[26] A. Davey, K. Stewartson, Proc. Roy. Soc. Lond. Ser. A 338101 (1974).

[27] T.B. Benjamin, J.E. Feir, J. Fluid Mech 27 417, (1967).

[28] V.E. Zakharov and L.A. Ostrovsky, Physica D, 238, 540, (2009).

[29] S. Vlasov, V. Petrishchev, and V. Talanov, Radiophys. Quantum Electronics 14 1062, (1970).

[30] C. Klein and N. Stoilov, Stud. Appl. Math., 238, 145, (2020).

[31] G. Biondini, G. Kovacic, J. Math. Phys., 55, 0310506, (2014).

[32] M. S. P. Eastham, The Spectral Theory of Differential Equations (Scottish Academic, Edinburgh, 1973).

[33] A.-K. Kassam and L. N. Trefethen, SIAM J. Sci. Comput., 261214 (2005).

[34] See Sections 1 and 2 of the Supplemental Material for details on numerical computations.

[35] See Sections 3-5 of the Supplemental Material for additional simulations. 